\title{
Environmental effects on the atomic gas content of galaxies in the local universe
}

\author{
Cheng Li \\ Max Planck Institute Partner Group at Shanghai Astronomical Observatory \\ Nandan Road 80, Shanghai 200030, China \\ email: leech@shao.ac.cn
}

\begin{abstract}
Effects of galaxy environment on its cold gas content are studied in detail using different date sets and statistics. These include measuring the clustering of galaxies as a function of their HI mass fraction, quantifying the depletion of HI gas content of cluster galaxies as a function of cluster-centric radius, and comparing the dependence of environmental density on galaxy star formation rate with the dependence on Hi gas mass fraction. Results from these studies are all consistent with a picture in which ram-pressure stripping may play an important role in removing atomic gas from the outer disks of low mass satellite galaxies.
\end{abstract}

Keywords. galaxies: abundances — galaxies: distances and redshifts — galaxies: formation galaxies: ISM — large-scale structure of universe — dark matter

\section{Introduction}

Gas-related "accretion" and "quenching" processes play crucial roles in galaxy formation. These processes are believed to be strongly connected with the environmental effects occurring within and around dark matter halos such as ram-pressure and tidal stripping. In order to better understand these effects, we have recently done a series of works based on large samples of low-redshift galaxies from recent optical photometric/spectroscopic and $\mathrm{HI}$ emission line surveys. These include measuring the clustering properties of galaxies as a function of their atomic gas mass fraction ( $\mathrm{Li}$ et al. 2012; hereafter L12), quantifying the depletion of atomic gas content of galaxies in/around clusters as a function of cluster-centric radius (Zhang et al. 2012; hereafter Z12), and examining the dependence of both atomic gas content and star formation rate on the local density of the galaxy environment (Fabello et al. 2012; hereafter F12).

Here we present our main results and refer the reader to the above papers for details.

\section{Galaxy biasing as a function of HI mass fraction}

We make use of large samples of galaxies selected from the Sloan Digital Sky Survey (SDSS; York et al. 2000) for our studies. The majority of our galaxies lack HI emission line observations, however. For this reason, in L12 we introduced a new photometric estimator of the HI mass fraction $\left(M_{H I} / M_{*}\right)$ in local galaxies. This is a linear combination of four parameters: stellar mass, stellar surface mass density, $N U V-r$ color, and $g-i$ color gradient, and is calibrated using samples of nearby galaxies $(0.025<z<0.05)$ with HI line detections from the GALEX Arecibo SDSS Survey (GASS; Catinella et al. 2010) and Arecibo Legacy Fast ALFA (ALFALFA; Giovanelli-05) surveys. When compared to previously determined estimators (e.g. Zhang et al. 2009; Catinella et al. 2010), the new estimator is demonstrated to provide unbiased $M_{H I} / M_{*}$ estimates even for HI-rich galaxies. This is shown in Fig. 1. 
We have applied this estimator to a sample of $\sim 24,000$ galaxies from the final data release of the SDSS. We bin these galaxies by stellar mass and HI mass fraction and compute projected two point cross-correlation functions with respect to a reference galaxy sample. Results are compared with predictions from current semi-analytic models of galaxy formation. The agreement is good for galaxies with stellar masses larger than $10^{10} M_{\odot}$, but not for lower mass systems. We then extend the analysis by studying the bias of HI-poor (upper panels of Fig. 2) or HI-rich (lower panels of Fig. 2) galaxies with respect to galaxies with normal Hi content on scales between $100 \mathrm{kpc}$ and $\sim 5 \mathrm{Mpc}$. For the HI-poor population, the strongest bias effects arise when the HI-deficiency is defined in comparison to galaxies of the same stellar mass and size. This is not reproduced by the semi-analytic models, where the quenching of star formation in satellites occurs by "starvation"; and does not depend on their internal structure. HI-rich galaxies with masses greater than $10^{10} M_{\odot}$ are found to be anti-biased compared to galaxies with "normal" HI content. Interestingly, no such effect is found for lower mass galaxies.
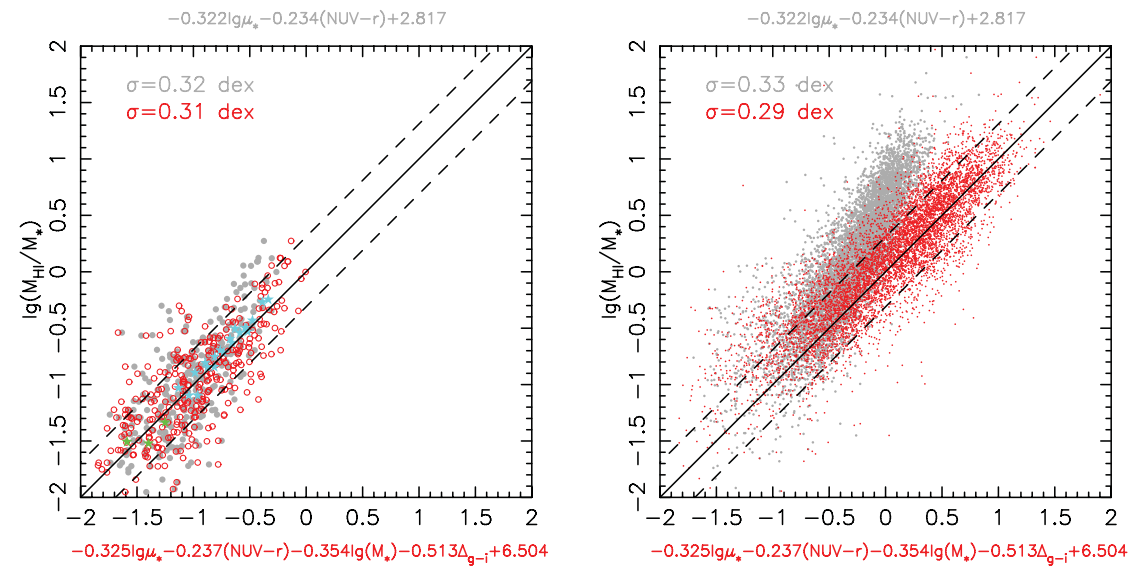

Figure 1. In the left-hand panel we plot in open circles the best-fitting relation between the $\mathrm{HI}$ mass fraction $\left(M_{H I} / M_{*}\right)$ and the linear combination of surface mass density $\mu_{*}, N U V-r$ color, stellar mass $M_{*}$ and the gradient in $g-i$ color $\left(\Delta_{g-i}\right)$ determined from the HI-detected galaxies in the GASS survey. The relation is given on the bottom of the panel. This is compared to the grey dots which show the relation obtained by Catinella et al. (2010) between $M_{H I} / M_{*}$ and the linear combination of $\mu_{*}$ and $N U V-r$ (given in grey on the top of the panel). Diamonds are results for the stacked spectra (the bottom three diamonds are for the three stacks on the red sequence). The right-hand panel shows the same thing for a sample of HI-rich galaxies from the ALFALFA survey. The solid and dashed lines in both panels indicate the $1: 1$ relation and $1 \sigma$ error region for the new estimator. Figure taken from Li et al. (2012)

\section{Gas depletion in cluster galaxies}

In Z12, we extended the work above by analyzing galaxies in 300 nearby groups and clusters identified in the SDSS using the photometric HI gas mass indicator of L12. We study the radial dependence of inferred gas mass fractions for galaxies of different stellar masses and stellar surface densities. At fixed clustercentric distance and at fixed stellar mass, lower density galaxies are more strongly depleted of their gas than higher density galaxies (Fig. 3). An analysis of depletion trends in the two-dimensional plane of stellar mass $M_{*}$ and stellar mass surface density $\mu_{*}$ reveals that gas depletion at fixed clustercentric radius is much more sensitive to the density of a galaxy than to its mass. This is consistent with that low density galaxies are more easily depleted of their gas, 

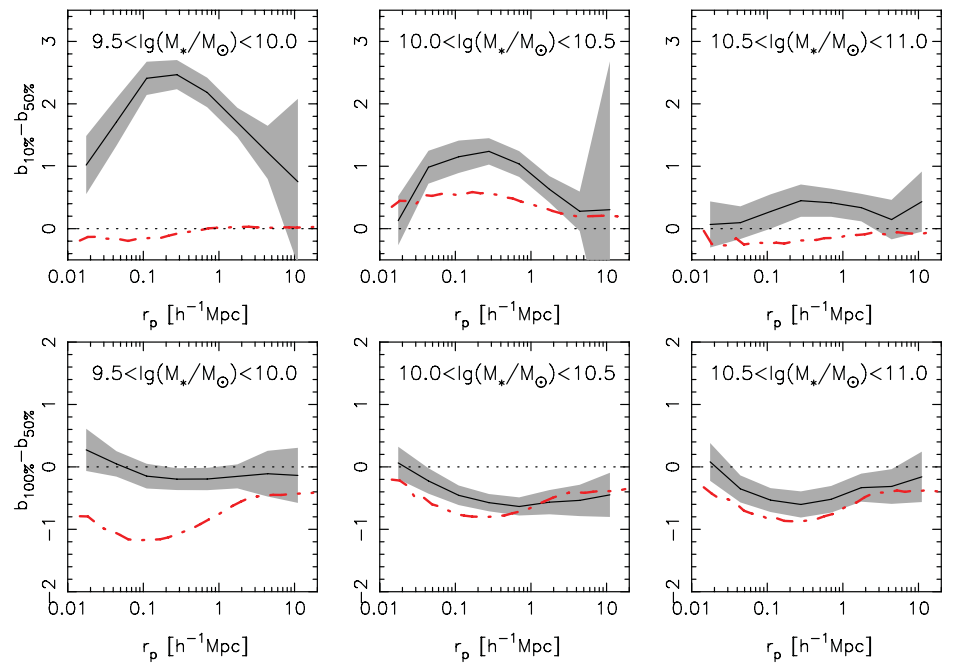

Figure 2. In the upper panels, we plot the change in bias factor from the $10^{\text {th }}$ percentile to the $50^{t h}$ percentile in $\log _{10}\left(M_{H I} / M_{*}\right)$ as a function of projected physical scale, for different stellar mass intervals as indicated. The solid line shows the result from the SDSS/DR7. The errors estimated from the mock catalogues are shown as shaded regions. The dotted-dashed line shows results from the semi-analytic model of $\mathrm{Fu}$ et al. (2010) after convolution with errors. The lower panels show the change in bias factor from the $50^{t h}$ to the $100^{t h}$ percentile. Figure taken from Li et al. (2012)

because they are more easily affected by ram-pressure and/or tidal forces. We also look at the dependence of our gas fraction/radius relations on the velocity dispersion of the cluster, finding no clear systematic trend.

\section{Dependence on local density}

We have also examined the environmental density dependence of the atomic gas mass fractions of nearby galaxies and compared this with the density dependence of their central and global specific star formation rates. Results are published in F12. In short, we stack Hi line spectra extracted from the ALFALFA survey centered on galaxies with UV imaging from GALEX and optical imaging/spectroscopy from SDSS. We use these stacked spectra to evaluate the mean atomic gas mass fraction of galaxies in bins of stellar mass and local density. For galaxies with stellar masses less than $10^{10.5} M_{\odot}$, the decline in mean atomic gas mass fraction with density is stronger than the decline in mean global and central specific star formation rate, as shown in Fig. 4. The same conclusion does not hold for more massive galaxies. We interpret our results as evidence for ram-pressure stripping of atomic gas from the outer disks of low mass satellite galaxies.

\section{Discussion}

Our results from L12 on galaxy clustering appear to suggest that, in the local universe, gas removal processes depend on the size or surface mass density of the galaxies. Thus our results indicate that processes such as ram-pressure stripping, which depend on the surface density of the interstellar medium, may play an important role in explaining the observed trends. If this was the case, we would also expect that lower mass galaxies is more likely to be affected by ram-pressure than higher mass galaxies, because the former 
have lower surface densities. In order to test this hypothesis in more detail, we have looked behavior of gas deficiency as a function of cluster-centric radius in samples of nearby groups and clusters (Z12), and found that the depletion of cold gas in galaxies depends not only on their mass, but also on their internal structure. This is well consistent with what we expected. Studies of the environmental densities of galaxies with different star formation rates and atomic mass fractions have also provided evidence in support of the ram-pressure effect to be a major role in removing the gas from galaxies.

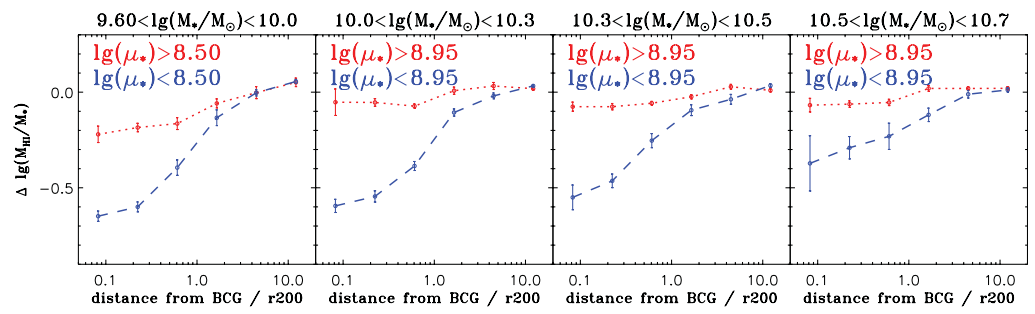

Figure 3. The difference in the median Hi mass fraction with respect to galaxies of the same stellar mass in the field is plotted as a function of distance from the brightest cluster galaxy. Results for different mass bins are shown in different panels, while the dotted/dashed lines in each panel shows the result for the subset with higher/lower surface mass density. Figure taken from Zhang et al. (2012)

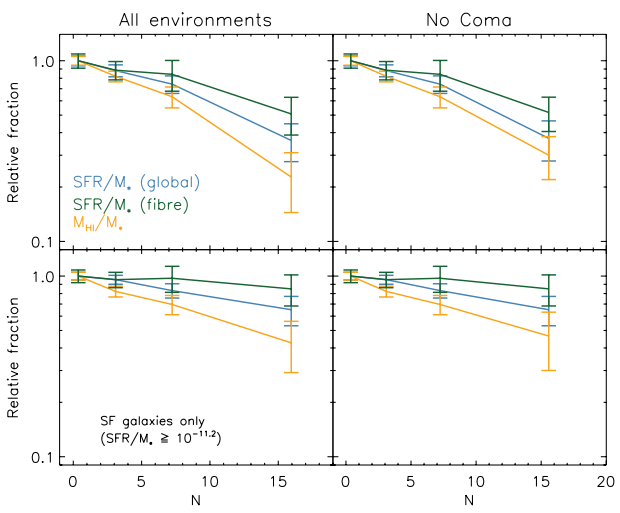

Figure 4. The relative dependence of Hi gas fraction (lowest line in each panel), the global specific star formation rate (middle line in each panel) and the fibre specific star formation rate (top line in each panel) as a function of local density for galaxies with $10<\log \mathrm{M}_{*}<10.5$. The left panels show results for all (top) or only the star forming ones (bottom) in our sample; the right panels show analogous results when galaxies in the vicinity of the Coma cluster are removed. Figure taken from Fabello et al. (2012)

\section{References}

Catinella, B., Schiminovich, D., Kauffmann, G., et al. 2010, MNRAS, 403, 683

Fabello, S., Kauffmann, G., Catinella, B., et al. 2012, arXiv:1209.2003

Giovanelli, R., Haynes, M. P., Kent, B. R., et al. 2005, AJ, 130, 2598

Li, C., Kauffmann, G., Fu, J., et al. 2012, MNRAS, 424, 1471

Zhang, W., Li, C., Kauffmann, G., et al. 2009, MNRAS, 397, 1243

Zhang, W., Li, C., Kauffmann, G., \& Xiao, T. 2012, arXiv:1207.3924

York, D. G., Adelman, J., Anderson, J. E., Jr., et al. 2000, AJ, 120, 1579 